\title{
Caractéristiques de la conduite des troupeaux allaitants en zones difficiles *
}

\author{
M Thériez, M Petit, W Martin-Rosset \\ INRA, centre de Clermont-Theix, 63122 Saint-Genès-Champanelle, France
}

(Reçu le 19 mars 1993 ; accepté le 22 décembre 1993)

\begin{abstract}
Résumé - Les troupeaux de bovins, d'ovins et d'équidés, élevés en milieux difficiles, sont soumis à différentes contraintes dont la principale est une période de faible disponibilité alimentaire de durée plus ou moins longue. Au cours de celle-ci, les animaux en stabulation sont souvent alimentés avec des fourrages de qualité médiocre à mauvaise, récoltés à un stade de maturité trop tardif. Ce type de régime entraîne des déficits alimentaires plus ou moins marqués selon la capacité de l'espéce à ingérer des aliments à forte valeur d'encombrement. Au pâturage les différences entre espèces sont moins marquées du fait des possibilités de tri. Cette période difficile entraîne une mobilisation des réserves corporelles, dont l'intensité dépend de la durée de la sous-alimentation, de l'état corporel initial et, surtout, du stade physiologique de la femelle, c'est-à-dire de ses besoins nutritionnels. Dans les 3 espéces étudiées, la productivité des troupeaux étant conditionnée par le niveau nutritionnel et par l'état corporel des femelles au moment de la mise à la reproduction et de la mise bas, il est nécessaire d'adapter les méthodes d'élevage pour permettre aux animaux de ne pas descendre en dessous d'etats minima. Ces adaptations portent sur le type de production (le plus souvent des jeunes qui sont vendus pour être engraissés dans des zones plus favorables), le choix des races et les systèmés de conduite. Les races adaptées à ces milieux se caractérisent par leur capacité à ingérer suffisamment de fourrages grossiers et par leur aptitude à utiliser et à reconstituer leurs réserves corporelles. Cela leur confère une capacité satisfaisante à produire régulièrement des jeunes et à les élever. La brièveté de la période d'alimentation abondante au pâturage limitant les possibilités de reconstitution des réserves corporelles, la première adaptation des systèmes de conduite aux conditions difficiles consiste à choisir des dates de reproduction et de mise bas qui permettent de faire coïncider ces périodes de besoins élevés avec celle de disponibilité alimentaire importante. La durée d'allaitement peut aussi être raccourcie.
\end{abstract}

femelle allaitante / zones difficiles / élevage

Summary - Management of suckling herds in harsh environments. Cattle, sheep and horses managed in harsh environments are submitted to various constraints including long or short periods of food scarcity. During these periods, animals are fed indoors with poor to bad quality roughages, cut at a late stage of maturity. Such diets induce nutritional deficits, of variable importance, according to the species ability to consume bulky feeds. Differences between species in this ability are re-

\footnotetext{
* Communication faite à la $7^{\mathrm{e}}$ Conférence sur la nutrition et l'alimentation des herbivores, Paris, 25-
} 26 mars 1992. Voir Ann Zootechn 42, 116 
duced at pasture, as a result of diet selection behaviour. The famine period induces a mobilisation of body reserves, whose intensity varies with the duration of the undernutrition period, the animal's initial body condition, and, above all, its physiologial state, ie its nutritional requirements. For the 3 species, productivity depends most on nutrition level and body condition at mating and parturition, therefore it is essential to adapt management procedures to prevent too low body condition scores at these critical times. Adaptations depend on the type of final product desired (most frequently lean young animals for fattening in more favoured areas), the breed of animals and management systems. The breeds adapled to harsh environments are characterised by their ability to consume large amounts of roughages and by their ability to mobilise and restore their body reserves. These abilities give them the capacity to produce and rear young on a regular basis. The shortness of the abundant grazing period limits possibilities for body reserve restoration, and therefore the first adaptation of management systems to harsh environments is to choose mating periods in order to synchronise periods of high nutritional requirements (end of pregnancy and lactation) with those of pasture lushness. An earlier weaning is also an efficient adaptation method.

\section{suckling herds / harsh environment / management}

\section{INTRODUCTION}

Les élevages allaitants, bovins, ovins et chevaux, ont un niveau de production biologique relativement faible, à quelques exceptions près (ovins en systèmes d'agnelages fréquents). II peuvent donc être exploités dans différents milieux, même peu productifs. En raison de la valeur marchande de leurs produits, ils sont de fait le plus souvent localisés dans les zones difficiles ou dans les parties les moins productives des exploitations.

Ces zones peuvent être caractérisées par leurs handicaps, soit climatique qui réduit souvent la période de végétation active à moins de 6 mois par an, soit pédologique ou topographique. Elles correspondent en France aux régions de montagne ou de certains piedmonts, aux zones méditerranéennes non irriguées, mais aussi à des zones de plaine aux sols pauvres et séchants. Plusieurs handicaps se cumulent souvent.

Dans beaucoup de ces zones, la seule ressource alimentaire est la végétation naturelle, disponible tout au long de l'année ou seulement pendant une période limitée, selon le climat et l'équilibre cheptelressource. Les troupeaux doivent couvrir leurs besoins alimentaires pendant plu- sieurs mois de l'année à partir de reports d'herbe sèche sur pied ou de fourrages récoltés, souvent en faible quantité ou de mauvaise qualité (faibles valeurs énergétique et azotée et forte valeur d'encombrement). Les conditions climatiques peuvent en outre aggraver la situation de l'animal, la chaleur, qui limite son appétit, ou le froid, qui augmente ses besoins d'entretien. Enfin, dans ces régions handicapées, les rendements des céréales restent faibles et les apports d'aliments concentrés achetés sont toujours limités pour des raisons économiques.

Ces troupeaux assurent une première fonction économique: la production de jeunes animaux sevrés qui sont le plus souvent vendus pour l'engraissement. La production d'animaux finis sur les exploitations est plutôt exceptionnelle dans les régions difficiles, à l'exception des agneaux et, parfois, des femelles réformées. Ces troupeaux remplissent également une seconde fonction, qui n'est pas nouvelle, mais dont l'importance et la prise de conscience émergent progressivement : par le pâturage et par la récolte des fourrages qui leur sont nécessaires, ils contribuent à l'entretien du paysage et à la gestion du territoire. Mais les règles de conduite de l'élevage pour assurer un 
entretien effectif du paysage ne sont pas toujours bien établies alors que celles de systèmes de production valorisant de telles surfaces ont fait l'objet de nombreuses études (Liénard et al, 1979).

\section{CONSÉQUENCES DU MILIEU SUR LE TROUPEAU}

\section{Utilisation des fourrages par les différentes espèces et conséquences sur la couverture de leurs besoins}

Les différentes espèces d'herbivores domestiques n'ont pas la même capacité à consommer des fourrages en quantité suffisante pour couvrir leurs besoins (Dulphy, 1994). Un fourrage à un stade végétatif donné permettra aux animaux auxquels il est distribué d'atteindre des niveaux nutritionnels variables selon leur type et leur stade physiologique. Et un même animal dans un état physiologique déterminé pourra couvrir ou non ses besoins alimentaires selon le stade de développement du fourrage qui lui est offert. Cette variabilité est illustrée par la figure 1 où sont reportés les bilans énergétiques d'une vache allaitante de $600 \mathrm{~kg}$ (Petit, 1998) d'une brebis de $60 \mathrm{~kg}$ ayant un seul agneau (Bocquier et al, 1988) et d'une jument lourde de $700 \mathrm{~kg}$ (INRA, 1990) nourries à volonté, mais à l'auge, c'est-à-dire avec une possibilité de tri limitée. Nous avons déterminé le taux de couverture des besoins énergétiques de chaque femelle, exprimé par rapport à ses besoins d'entretien, à 3 périodes types de son cycle de production (repos ou début de la gestation, fin de la gestation et pic de la lactation), et selon le stade végétatif d'un même fourrage vert (prairie de plaine aux stades pâturage, épiaison et floraison). Quel que soit leur stade physiologique, les juments sont toujours en bilan positif. Les vaches ne sont en bilan négatif qu'en fin de gestation et en début de lacta-
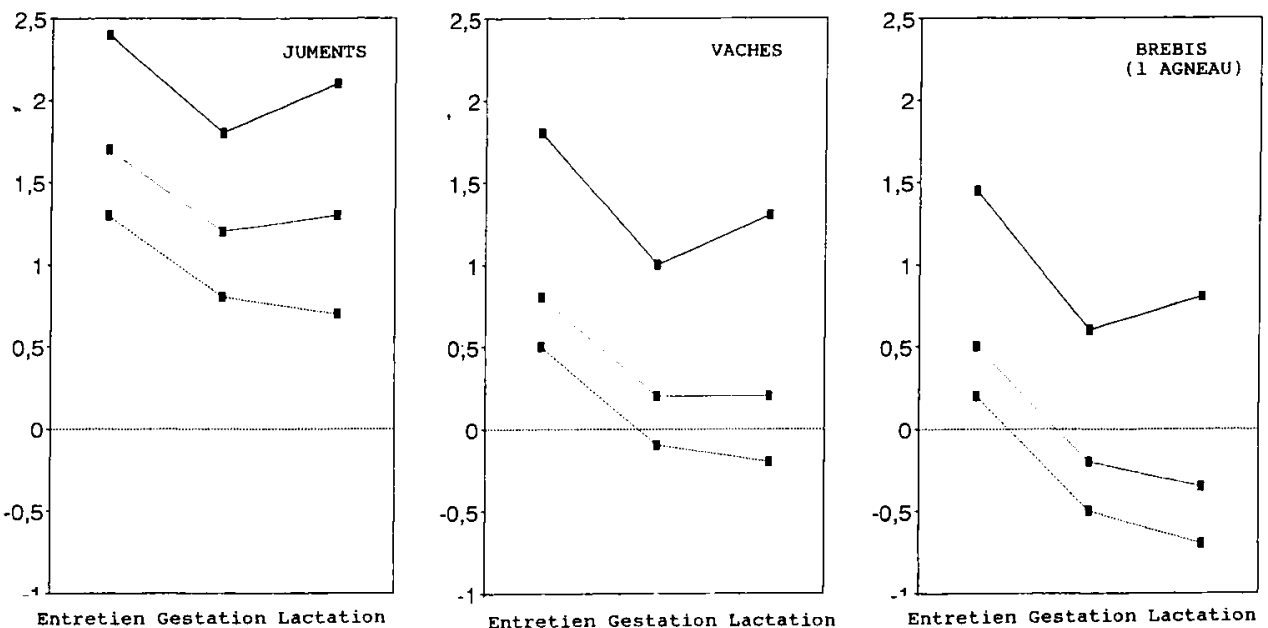

Fig 1. Bilan énergétique de juments, vaches et brebis selon le stade du fourrage (besoins exprimés en multiples du besoin d'entretien, herbe distribuée à l'auge). —— prairie naturelle au stade pâturage ; … $n$... prairie naturelle au stade épiaison :--__- prairie naturelle au stade floraison. 
tion lorsqu'elles reçoivent de l'herbe au stade floraison. Les brebis ne peuvent plus couvrir leurs besoins de fin de gestation et d'allaitement dès que l'herbe atteint le stade épiaison.

En hiver, ces mêmes animaux disposent de foins de moindre valeur alimentaire que le fourrage vert initial. Un bilan analogue est présenté sur la figure 2 avec de l'herbe au stade floraison, offerte à l'auge en vert ou après fanage dans de bonnes conditions. Vaches et juments taries sont en bilan positif quel que soit le mode de présentation et de conservation alors que les brebis taries nourries avec le foin coupé au stade floraison sont déjà en déficit (modéré). Avec ce même foin tardif seules les juments sont capables de couvrir leurs besoins de fin de gestation et de début de lactation.

Les conséquences d'une alimentation à base exclusive de foin de qualité moyenne ou médiocre seront donc différentes pour ces 3 espèces, la brebis devant utiliser de manière beaucoup plus importante ses réserves corporelles.
Mais en condition réelle de pâturage, la situation est sensiblement différente du fait du comportement de tri différent des espèces animales (Milne, 1994). Sur prairie herbacée, les ovins exercent une sélection très forte et la valeur alimentaire de leur régime est beaucoup plus élevée que celle de l'herbe disponible (fig 3 ; INRA, 1979). Les bovins, qui ont une capacité d'ingestion plus élevée, exercent un tri moindre que les brebis, et les juments prélèvent une ration presque représentative du tapis végétal offert. Le comportement de tri est plus marqué dans les 3 espèces sur des végétations hétérogènes (Loiseau et Martin-Rosset, 1989), mais les différences de comportement se maintiennent.

Ce comportement de tri permet aux différents herbivores d'accroître les quantités d'éléments nutritifs tirés du pâturage, et ainsi de reconstituer plus aisément leurs réserves corporelles utilisées pendant les périodes de sous-nutrition.

Dans la majorité des cas, les milieux difficiles se distinguent des zones favorables, non par la qualité des régimes ingérés au
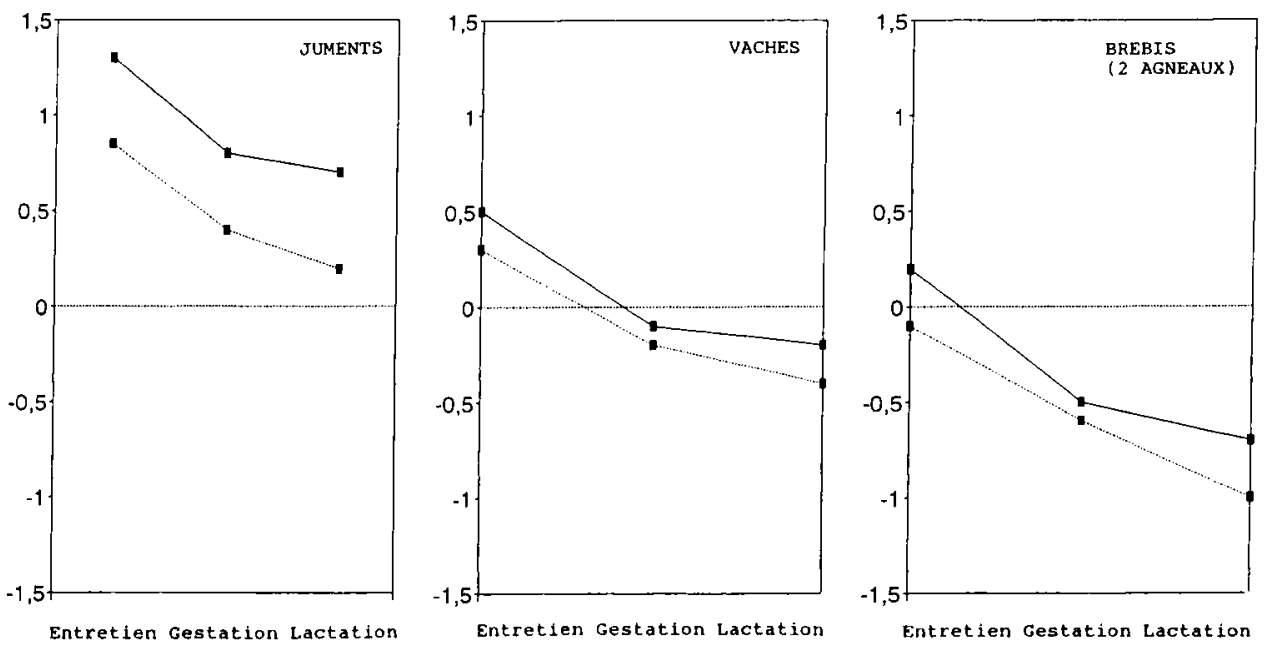

Fig 2. Bilan énergétique de juments, vaches et brebis recevant à l'auge une herbe coupée au stade début floraison ou la même herbe après fanage par beau temps. - - fourrage vert ; - 


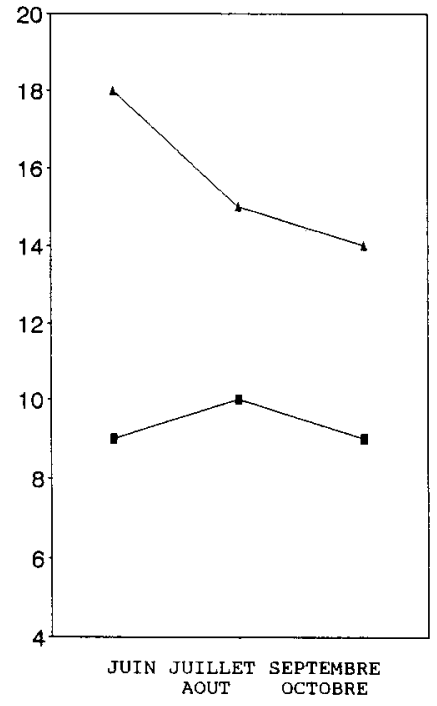

Fig 3. Effets du tri sur la qualité du régime de brebis sur une Nardaie dans le Massif central. - - teneur en MAT du fourrage sur pied: - $\mathbf{A}$ - teneur en MAT du régime.

pâturage, mais par la brièveté de la période favorable. Leur exploitation nécessite donc une adaptation des systèmes d'élevage à cette courte durée.

\section{Le cycle de production : variations de l'état corporel et conséquences}

Au cours d'un cycle de production les besoins alimentaires d'une femelle varient fortement, comme cela est reporté sur la figure 4 (Petit, 1988 ; Bocquier et al, 1988 ; INRA, 1990) où ils sont exprimés par rapport aux besoins d'entretien de l'espèce.

Dans les conditions pratiques de l'élevage les apports alimentaires sont rarement égaux à ces besoins. Ils leur sont le plus souvent inférieurs pendant les phases d'alimentation en bâtiments avec des four- rages conservés de qualité médiocre ou au pâturage pendant la période d'arrêt de la végétation. Les femelles allaitantes doivent donc alors faire appel à leurs réserves corporelles : réserves protéiques et minérales en quantités limitées et surtout réserves lipidiques. Cette utilisation des réserves entraîne un amaigrissement de l'animal dont le poids vif et l'état corporel varient au cours du cycle de production (tableau I).

Les conséquences des périodes de sous-alimentation dépendent de l'ampleur du déficit, du stade physiologique de l'animal et des caractéristiques de l'espèce. II est souhaitable à ce titre de séparer les vaches et juments des brebis. Les premières mettent bas et allaitent le plus souvent un seul jeune. Le poids à la naissance et la croissance ultérieure de ce jeune varient peu en cas de sousalimentation même assez sévère de sa mère, sous réserve que celle-ci soit en bon état au début de la période de sousalimentation. En revanche, la femelle devant être fécondée de nouveau en début d'allaitement pour maintenir le rythme de reproduction d'une mise bas par an, le déficit tolérable est alors limité si la période de reproduction débute avant la mise à l'herbe de printemps (Petit, 1988; Bocquier, 1988 ; INRA, 1990).

Inversement les portées multiples sont fréquentes chez les ovins, même dans les troupeaux de zones défavorisées et les brebis portant deux fœtus ou plus ne peuvent pas compenser totalement le déficit alimentaire en fin de gestation. Ceci se traduit alors par des troubles graves : toxémie de gestation pouvant entraîner l'avortement et même la mort de la mère ou une forte mortalité néonatale des agneaux. En revanche, la gestation de la brebis ne durant que 5 mois, il est possible de la faire maigrir fortement en début de lactation sans effet négatif sur son rythme de reproduction ou sa prolificité car elle peut reconstituer ses réserves corporelles au cours 

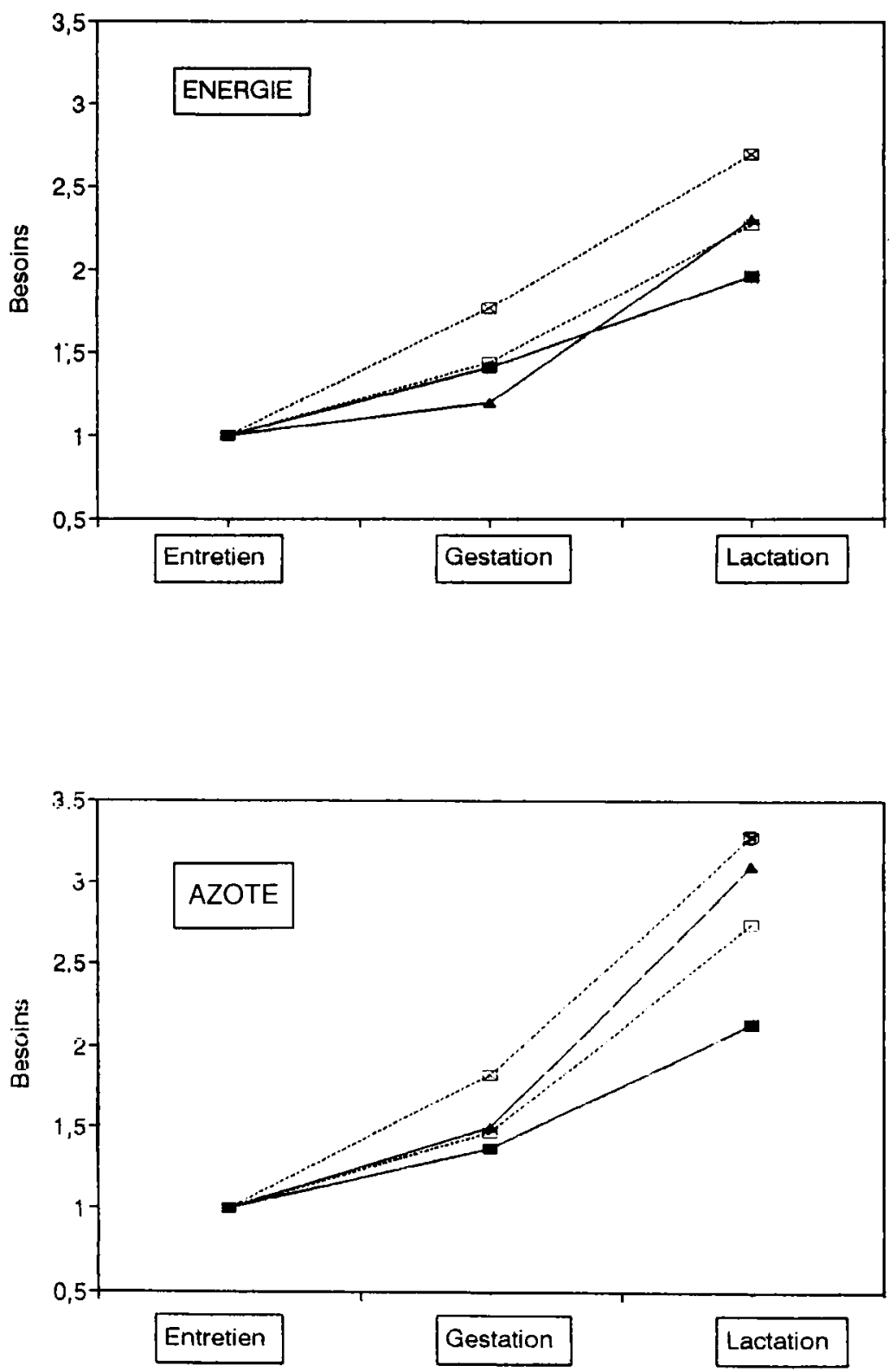

Fig 4. Variation des besoins énergétiques et azotés de juments, vaches et brebis selon leur stade physiologique (besoins exprimés en multiples du besoins d'entretien). $-\square$-- brebis à 1 agneau ; -- Х-- brebis à 2 agneaux ; $\square-$ vache ; $-\boldsymbol{\Delta}-$ jument. 
Tableau I. Utilisation des réserves corporelles dans les différentes espèces selon l'état physiologique.

$\begin{array}{llc}\text { Brebis a } & \text { Vache }^{\mathrm{b}} & \text { Jument } \\ \text { (UFL) } & \text { (UFL) } & \text { (UFC) }\end{array}$

$\begin{array}{lccc}\begin{array}{l}\text { Épargne énergétique correspondant } \\ \text { à la perte d'1 kg de poids vif }\end{array} & 2 \text { à } 15 & \begin{array}{c}3 \text { à } 7 \\ (5)\end{array} & 2 \text { à } 4 \\ \begin{array}{l}\text { Épargne énergétique correspondant } \\ \text { à la perte d'1 point de note d'état }\end{array} & 25 & \begin{array}{c}150 \text { à } 230 \\ (190)\end{array} & 110 \text { à } 170\end{array}$

a Variations dues aux différences de contenu digestif en début de lactation selon la qualité du fourrage. ${ }^{b}$ Résultats de différentes expériences; entre parenthèses, valeur moyenne retenue.

des 3 à 4 mois de repos après tarissement (Bocquier et al, 1988).

Une bonne gestion des réserves corporelles a pour objectif essentiel l'obtention d'un taux élevé de reproduction malgré des périodes plus ou moins longues de sous-alimentation. D'où les limites tolérables (et économiquement recommandées) des déficits énergétiques indiqués au tableau II. Ces limites, qui diffèrent selon le stade physiologique et l'espèce, supposent en outre que les femelles sont en bon état avant la période de restriction.

Tableau II. Écarts maxima entre besoins énergétiques et apports alimentaires.

\begin{tabular}{lccc}
\hline Stade & \multicolumn{3}{c}{$\begin{array}{c}\text { Espèce } \\
\end{array}$} \\
& Brebis * Vache** Jument & \\
& & & \\
\hline & & & \\
Fin de gestation & 0,20 & 0,45 & 0,25 \\
Début de lactation & 0,85 & 0,25 & 0,15 \\
Période reproduction & 0 & 0 & - \\
\hline
\end{tabular}

Écarts exprimés par rapport aux besoins d'entretien. - D'après Bocquier et al (1988); ** d'après Petit (1988); *** d'après INRA (1990).
Le résultat de la période de reproduction dépend fortement de l'état corporel de l'animal et du niveau des apports alimentaires qui lui sont alors offerts (tableau III). Une reprise rapide des cycles cestriens des femelles en lactation (vache, jument et brebis en système d'agnelage accéléré) et un taux d'ovulation élevé (brebis) ne peuvent être obtenus que si l'état corporel de ces femelles ne descend pas en dessous d'un niveau seuil à la période clef du cycle (tableau IV ; Petit, 1988 ; Bocquier et al, 1988; INRA, 1990). Dans les troupeaux de vaches allaitantes, cette période de référence est le vêlage. L'intervalle vêlagepremier œstrus est en étroite relation avec l'état corporel à la mise bas. Celui-ci doit, dans le cas de vêlages de début et milieu d'hiver, être supérieur ou égal à 2,5 (Petit, 1988), ce qui évite d'atteindre un niveau trop faible après 2 à 3 mois de sousalimentation en lactation. Pour la jument qui est capable d'augmenter très rapidement sa consommation et de reconstituter des réserves corporelles en début de lactation, même parfois aux dépens de sa production laitière (Martin-Rosset et Doreau, 1984 ; Doreau et al, 1988), la période de référence est le tout premier mois de lactation, qui correspond à la reproduc- 
Tableau III. Effets du niveau alimentaire ou de l'état corporel sur la reproduction des femelles allaitantes.

\section{Effet du niveau alimentaire}

$\begin{array}{lcc}\text { Juments en bon état corporel initial } & & \\ \text { Taux de couverture } & & 80 \\ \text { des besoins énergétiques (\%) } & 100 & 16,1 \\ \text { Intervalle mise bas-ovulation (j) } & 17,6 & 64 \\ \text { Juments gestantes } & 77 & 11 \\ \text { sur le } 1^{\text {er œstrus (\%) }} & 15 & 82 \\ \text { sur le } 2^{\mathrm{e}} \text { œstrus (\%) } & 92 & \end{array}$

Brebis sur nardaie en lutte de juin

Chargement.(brebis/ha)

Fertilité (\%)

Prolificité (agneau/mise bas)

Productivité (agneau/brebis)

$\begin{array}{cc}5 & 10 \\ 87 & 80 \\ 1,6 & 1,5 \\ 1,4 & 1,2\end{array}$

Effet de l'état corporel et du niveau alimentaire

Vaches allaitantes à 2 états corporels initiaux

État corporel au vêlage

Régime alimentaire

Vaches cyclées $50 \mathrm{j}$ après vêlage (\%)

Vaches gestantes 5 mois après vêlage (\%)

$\begin{array}{cccc}1,5 & & 3,0 & \\ \text { Haut } & \text { Bas } & \text { Haut } & \text { Bas } \\ 27 & 38 & 63 & 42 \\ 91 & 70 & 91 & 85\end{array}$

Tableau IV. Notes d'état corporel recommandé à différents stades.

\begin{tabular}{|c|c|c|}
\hline \multirow[t]{2}{*}{ Espèce } & \multicolumn{2}{|c|}{ Stade physiologique } \\
\hline & Mise à la saillie & Mise bas \\
\hline Vache & 1,5 à 2,5 & 2,5 à 3 \\
\hline Brebis & 2,5 a 3 & 3 à 4 \\
\hline Jument & 2,5 & 2,5 \\
\hline
\end{tabular}

tion. Chez la brebis, c'est l'état au début de la période de lutte qui est important pour maintenir une prolificité suffisante.

II est possible de corriger en partie les effets d'un état corporel légèrement insuffisant, par une suralimentation temporaire.
Cette technique, connue sous le nom de "flushing", est couramment pratiquée chez les brebis pour améliorer leur prolificité. Elle est inefficace chez des animaux trop maigres, dont l'état corporel est inférieur à 2. Pratiqué chez la vache allaitante, selon la même technique que sur la vache traite, le flushing semble le plus souvent inefficace. Cependant la mise à l'herbe en conditions favorables permet un retour rapide en chaleurs des vaches maigres après vêlage (note $<1,5$ ). Cette technique n'est pas utilisée sur les juments car celles-ci sont capables de consommer en début de lactation 1,5 à 2 fois plus de matière sèche qu'en fin de gestation (Boulot, 1987 ; Doreau et al, 1991). 


\section{ADAPTATION DES SYSTĖMES D'ÉLEVAGE}

Face aux contraintes de nature diverse auxquelles ils sont confrontés, les éleveurs doivent adapter leurs systèmes d'élevage pour en assurer la pérennité. Cette adaptation revêt différents aspects dont les principaux concernent le maintien de ressources alimentaires de qualité, le choix des races, la pratique de déficits alimentaires tolérables et enfin l'ajustement des périodes de besoins élevés à celles d'abondance alimentaire.

\section{L'amélioration de la qualité des ressources fourragères}

Les éleveurs y ont recours de différentes façons. Pour mémoire, rappelons qu'il est de pratique courante d'adjoindre aux ressources naturelles des fourrages cultivés (en quantité variable) sur les meilleures parcelles de l'exploitation, pour accroître les ressources en période de disette (ressources hivernales, repousses d'été, etc). Cela peut permettre aussi d'améliorer la qualité du régime alimentaire quotidien des animaux pâturant en les faisant passer en un temps limité sur des prairies de très bonne qualité, comme cela est pratiqué dans les troupeaux gardiennés dont le circuit quotidien se termine sur une prairie artificielle (Meuret, 1993).

L'amélioration de la flore naturelle d'une partie plus ou moins importante de l'exploitation est également pratiquée selon différentes méthodes parmi lesquelles on peut citer :

- le sursemis de nouvelles espèces (dont les Légumineuses, notamment sur les parcours méditerranéens), qui améliore la qualité des fourrages disponibles, assure en outre une production plus précoce et plus abondante au printemps ;
- le parcage de nuit qui permet un transfert de fertilité des zones les moins favorables vers celles potentiellement plus productives. Utilisé depuis très longtemps mais exigeant en main d'cuvre, il est de moins en moins pratiqué, malgré son efficacité ;

- l'introduction, à côté de l'espèce animale principale exploitée, d'autres espèces capables de maîtriser certains végétaux envahissants comme la bruyère, les genêts ou la myrtille en zone de montagne ou le chêne kermès en garrigue (INRA, 1979 ; Loiseau et Martin-Rosset, 1989) est
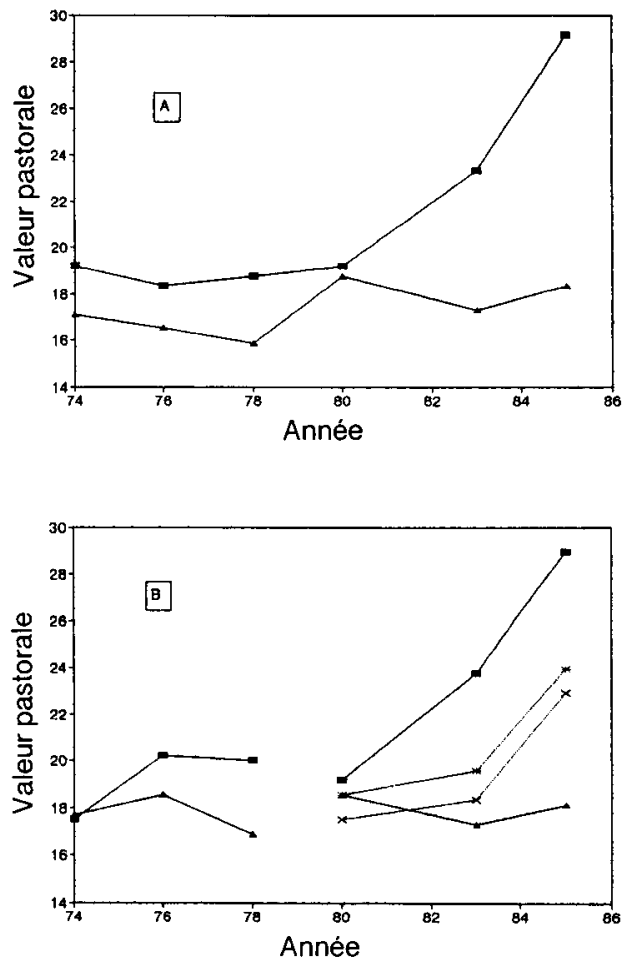

Fig 5. Évolution comparée de l'indice moyen de valeur pastorale d'un pâturage de montagne (Auvergne, $1400 \mathrm{~m}$ d'altitude) exploité par des chevaux et (ou) des ruminants. D'après Loiseau et Martin-Rosset (1989). A : Comparaison chevaux-bovins : $\longrightarrow$ - chevaux ; $-\mathbf{\Delta}-$ bovins. $\mathrm{B}$ : pâturage mixte :- chevaux ; --vaux + moutons ; $-\mathbf{A}-$ bovins ; -- $x--$ bovins + moutons. 
également pratiquée. Le cheval est à ce titre un moyen d'action efficace, comme l'a montré une série d'expériences poursuivies pendant 12 ans sur des pâturages d'altitude des monts Dore (fig 5). L'amélioration de la valeur pastorale de ces pâturages se manifeste par une forte réduction de la contribution spécifique des ligneux (myrtille, genêt et bruyère) et des Graminées de la lande (nard en particulier) et par une augmentation de celle des Graminées fourragères (fétuque et agrostis). Le pâturage mixte avec chevaux doit être poursuivi après la phase d'amélioration pour assurer l'entretien des surfaces exploitées.

\section{Le choix des races}

Les éleveurs des zones difficiles ont sélectionné des races adaptées à leur milieu, désignées sous le terme de "races de pays" ou "races rustiques", sans que ces termes correspondent à une qualification très précise. Ces races présentent un certain nombre de caractéristiques communes et en particulier de bonnes aptitudes maternelles, c'est-à-dire la capacité de produire facilement et régulièrement des jeunes qu'elles élèvent en ces milieux difficiles. Ces qualités maternelles dépendent directement de leur aptitude à ingérer suffisamment de fourrages, pour réduire le déficit alimentaire au cours des périodes de disette (hivernale par exemple) et pour récupérer rapidement leur état corporel au cours des périodes plus libérales (le plus souvent en pâturage de printemps). Ces races disposent en outre de réserves corporelles suffisantes qui leur permettent de passer les caps difficiles sans pénaliser leur production laitière et surtout leur reproduction. Elles présentent par contre le plus souvent, chez les ovins comme chez les bovins, un défaut considéré comme majeur, une conformation bouchère moyenne à médiocre qui pénalise leurs produits à la vente, défaut qui peut être réduit par la pratique du croisement industriel sur une partie du cheptel (Bibe et Vissac, 1979).

Ces aptitudes sont liées à un certain nombre de caractéristiques communes dont la précocité de développement est un élément important qui permet aux jeunes femelles rustiques d'atteindre plus rapidement et plus aisément leur "état adulte» et de disposer, à un même âge, de plus de tissus adipeux que leurs homologues de races plus tardives. Cela les rend moins fragiles lors de leurs premiers cycles de production. Un grand format peut être un élément favorable (plus de réserves), aussi bien pour les bovins et les ovins que pour les chevaux, mais il peut aussi être associé à une plus lente obtention du poids "adulte". II existe en fait des races rustiques de petit format dans les 3 espèces, dont en France l'Aubrac pour les bovins, la Charmoise ou la Mérinos d'Arles pour les ovins, la Merens pour les chevaux.

Pour illustrer ces différences entre races, nous avons reporté sur le tableau $V$ les tout premiers résultats de la comparaison à Marcenat (1 000-1 $100 \mathrm{~m}$ ), dans le Cantal, de 2 races très différentes par leurs aptitudes : la Salers et la Limousine. Les Salers, de format un peu plus grand et de plus forte capacité d'ingestion que les Limousines, ont les croissances les plus élevées au cours de la phase d'élevage, tant en hiver qu'au pâturage et pèsent à 30 mois $80 \mathrm{~kg}$ de plus que les Limousines élevées dans les mêmes conditions. Ces différences se maintiennent après le premier vêlage, tant pour la consommation de fourrages conservés que pour la reprise de poids au pâturage. Les veaux des Salers, meilleures laitières, pèsent $282 \mathrm{~kg}$ à 8 mois contre $266 \mathrm{~kg}$ pour les Limousins malgré la consommation de 150 à $200 \mathrm{~kg}$ environ de concentré par ces derniers. Les Limousines vêlent, en moyenne, 2 semaines plus tard que les Salers, retard qui pourrait être une forme d'adaptation au mi- 
Tableau V. Comparaison entre des vaches Salers et Limousines.

\begin{tabular}{lll}
\hline Race & Salers & Limousines \\
& & \\
\hline $\begin{array}{l}\text { Génisses d'élevage } \\
\text { Quantité de MS ingérée en hiver a } \\
\text { Gain de poids vif }(\mathrm{kg} / \mathrm{j})\end{array}$ & 1,66 & 1,52 \\
$\quad$ & 0,52 & 0,32 \\
$\quad$ En hiver & 0,60 & 0,46 \\
$\quad$ Au pâturage à un an & 584 & 505 \\
Poids à 30 mois $(\mathrm{kg})$ & & \\
& & 1,4 à 2,0 \\
Vaches adultes & 1,7 à 2,2 & $25 / 02$ \\
Quantité de MS ingérée en hiver b & $10 / 02$ & 600 \\
Date moyenne de vêlage & 645 & 49 \\
Poids après vêlage $(\mathrm{kg})$ & 72 & 237 à 266 \\
Reprise de poids au pâturage $(\mathrm{kg})$ & 282 & 0 à 200 \\
Poids des veaux à 8 mois $(\mathrm{kg})$ & 0 & \\
Concentré par veau $(\mathrm{kg})$ & & \\
\hline
\end{tabular}

a Valeurs exprimées en $\%$ du poids vif $^{b}$ valeurs exprimees en $\%$ du poids vit, pendant la gestation puis en lactation.

lieu comme cela avait déjà été observé dans l'étude "Ranch ITEB-INRA" entre 1971 et 1976.

D'autres caractères peuvent différencier les races sur leur aptitude à valoriser les milieux difficiles. Ainsi, par exemple, les brebis Mérinos d'Arles seraient mieux adaptées à la transhumance que celles d'autres races exploitant avec elles les Alpes du Sud. Cette meilleure adaptation serait due pour partie à leur comportement grégaire qui facilite la surveillance et la conduite du troupeau, ainsi qu'au développement de leur toison. Soumises à la pluie et au vent en chambre respiratoire, leurs pertes thermiques à $15^{\circ} \mathrm{C}$ sont de $78 \%$ inférieures à celles des Préalpes (Hocquette et al, 1992).

\section{L'utilisation des réserves corporelles}

Les variations de l'état corporel d'une femelle évoquées précédemment (tableau
IV) sont "normales» et dans certaines limites sans conséquences sensibles sur sa production et sa carrière si, au cours de chaque cycle, des phases d'alimentation libérale lui permettent de reconstituer les réserves utilisées en période de pénurie. Dans un milieu donné, le niveau de mobilisation tolérable est donc pour beaucoup déterminé par les possibilités de reconstitution. Les élevages en zone de montagne, qui ont la période de pénurie la plus longue, ont aussi la période de reconstitution au pâturage la plus courte (tableau VI). Dans ces zones, toutes choses égales par ailleurs, l'utilisation des réserves corporelles devrait donc rester plus faible que dans les régions de plaine.

\section{Le choix des dates de reproduction}

L'éleveur dispose enfin de 2 moyens, très bien connus depuis longtemps des zootechniciens, pour adapter son système 
Tableau VI. Limites de la reconstitution des réserves corporelles de vaches Charollaises selon la durée de pâturage.

\begin{tabular}{lcc}
\hline Localisation & $\begin{array}{c}\text { Durée de } \\
\text { la saison } \\
\text { de páturage } \\
\text { (j) }\end{array}$ & $\begin{array}{c}\text { Gain de } \\
\text { poids vif } \\
\text { possible } \\
(\mathrm{kg})\end{array}$ \\
\hline Plaine (Normandie) & 195 & 92 \\
$\begin{array}{l}\text { Moyenne montagne (1 050 m) } \\
\text { Montagne (1 350 m) }\end{array}$ & 160 & 72 \\
& 135 & 58 \\
\hline
\end{tabular}

d'élevage aux ressources fourragères: le choix de la période de reproduction et de la date de sevrage. La première lui permet de situer le stade de besoins élevés qu'il estime prioritaire (allaitement et/ou saillie du cycle suivant) pendant la phase d'abondance des ressources alimentaires. Le choix de la date de sevrage permet de dissocier plus ou moins tôt le couple mère/ jeune. Dans le cas de la production d'agneaux au pâturage, le sevrage permet de favoriser le jeune aux dépens de sa mère lorsque la concurrence entre eux de- vient excessive et d'améliorer ainsi la croissance de l'agneau.

L'espèce ovine, du fait de la séparation le plus souvent complète entre les phases gestation et lactation et de la présence d'une période de "repos" total entre 2 cycles annuels successifs, est celle qui offre la plus grande possibilité de choix des dates de reproduction, surtout lorsque la race utilisée a une saison de reproduction de longue durée ( 8 mois ou plus). Cette possibilité est illustrée sur le tableau VII sur lequel on observe que la date d'agnelage de printemps à l'extérieur est d'autant plus avancée que la période de croissance de la végétation est précoce. On passe ainsi d'agnelages d'avril dans des régions à printemps tardif comme l'Écosse, à des mises bas de février en zone méditerranéenne à hiver froid comme le sud de la France, voire à des agnelages de fin d'automne ou d'hiver en Afrique du Nord pour disposer du pâturage pendant la lactation et finir les agneaux avant les fortes chaleurs.

Lorsque l'agnelage est réalisé en bergerie, il est possible d'anticiper sur la croissance de l'herbe et de commencer la lacta-

Tableau VII. Systèmes d'élevage ovin dans différentes zones difficiles.

\begin{tabular}{llcl}
\hline Région & $\begin{array}{l}\text { Croissance active } \\
\text { de la végétation }\end{array}$ & $\begin{array}{c}\text { Durée de la } \\
\text { saison sexuelle }\end{array}$ & $\begin{array}{c}\text { Période } \\
\text { d'agnelage }\end{array}$ \\
\hline $\begin{array}{l}\text { Écosse } \\
\text { Centre-Ouest }\end{array}$ & $\begin{array}{l}\text { avril-août } \\
\text { mars-juin } \\
\text { + septembre } \\
\text { avril-juillet } \\
\text { + septembre } \\
\text { février-mai } \\
\text { Massif central }\end{array}$ & $\begin{array}{l}4 \text { mois } \\
6 \text { mois }\end{array}$ & $\begin{array}{l}\text { avril (extérieur) } \\
\text { février (bergerie) }\end{array}$ \\
$\begin{array}{l}\text { Garrigues } \\
\text { (sédentaires) }\end{array}$ & 8 mois & mars (bergerie) \\
$\begin{array}{c}\text { Provence } \\
\text { (transhumants) }\end{array}$ & $\begin{array}{l}\text { + juin-sept } \\
\text { hiver-printemps }\end{array}$ & 8 mois & février (extérieur) \\
\begin{tabular}{l} 
Afrique du Nord \\
\hline
\end{tabular} & 10 mois & octobre (extérieur) \\
\hline
\end{tabular}


tion avec des fourrages conservés. Cette méthode permet d'accroître la proportion d'agneaux élevés à l'herbe avant l'été. Ceux-ci, âgés de 4 à 6 sem à la mise à l'herbe, sont plus aptes à consommer l'herbe de printemps et plus résistants aux conditions climatiques difficiles. Les besoins du couple mère-agneau étant alors maxima, il est plus facile de maîtriser la croissance de l'herbe. Cette situation est celle du Centre-Ouest ou des éleveurs herbagers du Massif central. Ces derniers disposent en outre, comme les éleveurs transhumants de Provence, des ressources alimentaires bon marché de la montagne pendant l'été. Leur système de production permet d'en tirer le meilleur parti, compte tenu des difficultés d'élever de jeunes agneaux à la montagne. L'augmentation des ressources de qualité est ici l'élément déterminant du système et de sa sécurité.

Le rythme de reproduction des troupeaux peut également dans certains cas, comme chez les éleveurs ovins du Massif central, concilier productivité élevée et conditions difficiles. Des agnelages tous les 8 à 9 mois, voire tout au long de l'année, permettent de sevrer plus de 1,6 agneau par brebis et par an sans recours excessif aux aliments concentrés car la proportion de portées de 3 agneaux ou plus, exigeantes du point de vue alimentation, reste très faible ( 5 à $6 \%$ ), très inférieure aux $35 \%$ observés dans un troupeau de productivité équivalente avec un seul agnelage par an (tableau VIII).

La réduction de la durée d'allaitement est également une adaptation des systèmes d'élevage aux milieux difficiles. Elle est actuellement largement utilisée par les moutonniers de ces zones qui ont saisi l'opportunité économique de l'exportation vers les pays méditerranéens (Espagne et Italie) d'agneaux de 22 à $24 \mathrm{~kg}$ de poids vif. Ils obtiennent sur ce marché un prix sensiblement voisin de celui des agneaux lourds d'au moins $35 \mathrm{~kg}$ qu'ils commercialisent en France.

Une telle adaptation des dates de mise bas et de durée d'allaitement se rencontre également en élevage bovin allaitant, et s'accompagne de changement d'époque et/ou d'âge au sevrage. Les 3 systèmes de production de veaux reportés sur le tableau IX, en cours d'expérimentation en montagne sur le domaine INRA de Laqueuille, se différencient par la date de vêlage : en début d'automne au pâturage, en hiver en stabulation, ou au printemps au pâturage. Dans le premier système, les

Tableau VIII. Composantes de la productivité selon le rythme de reproduction.

\begin{tabular}{|c|c|c|c|c|c|c|}
\hline \multirow[t]{2}{*}{ Système } & \multirow[t]{2}{*}{ Productivité a } & \multirow[t]{2}{*}{ Agnelages ${ }^{b}$} & \multirow[t]{2}{*}{ Prolificitéc } & \multicolumn{3}{|c|}{ Proportion } \\
\hline & & & & $S$ & $D$ & $T$ \\
\hline 3 en 2 ans & 1,64 agn & $1,32 \mathrm{mb}$ & $1,39 \mathrm{agn} / \mathrm{mb}$ & 46 & 49 & 5 \\
\hline 3 en 2 ans & 1,79 agn & $1,40 \mathrm{mb}$ & $1,43 \mathrm{agn} / \mathrm{mb}$ & 42 & 52 & 6 \\
\hline 1 par an & 1,94 agn & $1,11 \mathrm{mb}$ & $1,96 \mathrm{agn} / \mathrm{mb}$ & 15 & 51 & 35 \\
\hline
\end{tabular}

\footnotetext{
a Nombre d'agneaux sevrés par brebis effectif de plus de 12 mois et par an. ${ }^{b}$ Nombre d'agnelages par brebis effectif de plus de 12 mois et par an. ${ }^{c}$ Nombre d'agneaux nés par agnelage, S: agneaux nés simples, D: agneaux issus de portées de 2, T: agneaux issus de portées de 3 agneaux ou plus. Les deux premières lignes sont les résultats observés dans un réseau de montagne de 13 exploitations (Liénard, communication personnelle), la première correspond a la moyenne observée, la seconde aux résultats d'un élevage très bien maîtrisé. La troisième ligne correspond à un troupeau expérimental INRA.
} 
Tableau IX. Époque de vêlage et performances d'un troupeau de Salers en montagne a.

\begin{tabular}{|c|c|c|c|}
\hline Période de vêlage & Automne & Hiver & Printemps \\
\hline Durée d'allaitement (mois) & 8,5 & 8,5 & 5,0 \\
\hline $\begin{array}{l}\text { Apports nécessaires (UFL) en hiver } \\
\text { États corporels cibles }\end{array}$ & 1750 & 1340 & 780 \\
\hline en début d'hiver & 2,5 & 3,5 & 2,5 \\
\hline en fin d'hiver & 1,5 & 2,5 & 1,5 \\
\hline \multicolumn{4}{|l|}{ Résultats } \\
\hline $\begin{array}{l}\text { Taux de gestation (\%) } \\
\text { (après } 2 \text { mois) }\end{array}$ & 87 & 83 & 89 \\
\hline Mortalité des veaux (\%) & $4-5$ & $6-8$ & $3-4$ \\
\hline Poids de veaux au sevrage $(\mathrm{kg})$ & 280 & 284 & 199 \\
\hline
\end{tabular}

a Laqueuille, 63; 1300 m d'altitude.

vaches sont en lactation durant tout l'hivernage, ce qui est coûteux en fourrages hivernaux dans les conditions de montagne; il correspond en fait aux régions à hiver court et à été sec; il permet en outre de disposer d'animaux à vendre à une période de faible disponibilité sur le marché. Le second système se rencontre actuellement dans la majorité des zones de production. Le troisième permet de réduire fortement les besoins en fourrages récoltés pour l'hivernage, pendant lequel les vaches sont taries et en milieu de gestation; il est très peu pratiqué en France, et s'apparente aux pratiques de "ranching" avec courte durée d'allaitement. II pourrait constituer en fait une solution de recours dans des régions à végétation précoce en saison mais d'assez courte durée. Les performances des mères (productivité au sevrage) sont proches dans les 3 systèmes, quoique légèrement inférieures en cas de vêlages d'hiver si la durée de la période de reproduction est limitée à 2 mois. Les 2 autres systèmes présentent en revanche des inconvénients : des apports hivernaux supplémentaires (ici +400 UFL environ) pour le veau né en automne, et un poids nettement plus faible au sevrage pour le veau né au printemps et allaité durant 5 mois seulement.

Chez le cheval lourd, le poulain peut peser de 320 à $380 \mathrm{~kg}$ au sevrage en automne selon sa date de naissance et les conditions de pâturage des poulinières (Martin-Rosset et Trillaud-Geyl, 1984). Dans les zones de montagne, les poulains doivent naitre un mois environ avant la date de mise à l'herbe pour atteindre 320 à $340 \mathrm{~kg}$ au sevrage à $190 \mathrm{j}$. Cette solution est un compromis entre une distribution très limitée de fourrages aux mères en hiver et l'exploitation presque exclusive de surfaces peu productives pendant l'été par le couple jument-poulain.

\section{CONCLUSION}

Les milieux d'élevage difficiles se caractérisent plus, quelle que soit l'origine de leur handicap, par la longue durée de la période de faible disponibilité fourragère que par la qualité de leurs ressources, exception faite des zones déjà fortement dégradées. Les systèmes d'élevage qui y 
sont pratiqués sont donc orientés essentiellement vers l'exploitation d'animaux à faibles besoins et/ou dont la période de besoins élevés est limitée dans le temps. Ce sont donc d'abord des systèmes producteurs de jeunes animaux vendus après sevrage. La seconde adaptation concerne le choix de périodes de reproduction faisant classiquement coïncider la période de besoins accrus du troupeau (et plus particulièrement du couple mèrejeune) avec la période de croissance de I'herbe, donc de disponibilité alimentaire importante, et ne conserver que des adultes aux besoins limités et des femelles de renouvellement pendant les périodes difficile. De tels systèmes ont entraîné la sélection d'animaux capables de supporter une sous-alimentation temporaire à ces périodes, sans conséquences sensibles sur leur performances, et de reconstituer rapidement leurs réserves corporelles lorsque les disponibilités alimentaires s'améliorent.

\section{RÉFÉRENCES}

Bibé B, Vissac B (1979) Amélioration génétique et utilisation de territoire. In : Utilisation par les ruminants des pâturages d'altitude et parcours méditerranéen ( $G$ Molenat, $R$ Jarrige, eds) INRA Publications, Versailles, 481-491

Bocquier $F$, Thériez $M$, Prache $S$, Brelurut $A$ (1988) Alimentation des ovins. In : Alimentation des bovins, des ovins et des caprins (R Jarrige, ed) INRA Publications, Versailles, 249-281

Boulot S (1987) L'ingestion chez la jument. Études de quelques facteurs de variation au cours du cycle gestation-lactation. Implications nutritionnelles et métaboliques. Thèse Université Rennes I ENSAR, 126 p

Doreau M, Boulot S, Martin-Rosset W (1988) Energy requirements and the feeding of mares during lactation. A review. Livest Prod Sci $20,53-68$

Doreau M, Moretti C, Martin-Rosset (1991) Effect of quality of hay given to mares round to foaling on their voluntary intake and foal growth. Ann Zootech 39, 125-131
Dulphy JP, Jouany JP, Martin-Rosset W, Thériez M (1994) Aptitude comparée de différentes espèces d'herbivores domestiques à ingérer et digérer des fourrages distribués à l'auge. Ann Zootech 43 (1), 11-32

Hocquette JF, Vermorel M, Bouix $J$ (1992) Influence du froid, du vent et de la pluie sur les dépenses énergétiques de sept types génétiques de brebis. Gen Sel Evol 24, 147-169

INRA (1979) Groupe de recherche sur les hauts pâturages dégradés des Mont-Dore. Aspects biologiques et techniques de la remise en exploitation des hauts pâturages dégradés des Monts-Dore. In : Utilisation par les ruminants des pâturages d'altitude et parcours méditerranéens ( $G$ Molenat, $R$ Jarrige, eds) INRA Publications, Versailles, 57-136

INRA (1990) Alimentation des chevaux (W Martin-Rosset, ed) INRA publications, Versailles, $232 \mathrm{p}$

Liénard $G$, Béranger $C$, Thériez $M$, MartinRosset W (1979) Choix des systèmes d'exploitation et des animaux utilisateurs des surfaces peu productives. In : Utilisation par les ruminants des paturages d'altitude et parcours méditerranéens (G Molenat, $R$ Jarrige, eds) INRA Publications, Versailles, 493-518

Loiseau P, Martin-Rosset W (1989) Évolution à long terme d'une lande de montagne pâturée par des bovins ou des chevaux. agronomie 9, 161-169

Martin-Rosset W, Doreau M (1984) Besoins et alimentation de la jument. In : Le Cheval (R Jarrige, W Martin-Rosset, eds) INRA Publications, Versailles, 209-238

Martin-Rosset W, Trillaud-Geyl C (1984) Exploitation du troupeau de juments lourdes allaitantes. In : Le Cheval (R Jarrige, W Martin-Rosset, eds) INRA Publication, Versailles, 209-238

Meuret M (1993) Piloter l'ingestion au pâturage. In : Pratiques d'élevage extensif. Identifier, modéliser, évaluer. INRA Études et recherches sur les sytèmes agraires et le développement 27, 161-198

Milne JA (1994) Comparative abilities of different herbivorous species to graze in upland areas: consequences for productivity and vegetation. Ann Zootech 43 (1), 3-9

Petit M (1988) Alimentation des vaches allaitantes. In : Alimentation des bovins, des ovins et des caprins (R Jarrige, ed) INRA Publications, Versailles, 158-184 